\title{
Article \\ A Multi-Core Controller for an Embedded AI System Supporting Parallel Recognition
}

\author{
Suyeon Jang, Hyun Woo Oh $\mathbb{D}^{\mathbb{C}}$, Young Hyun Yoon, Dong Hyun Hwang, Won Sik Jeong and Seung Eun Lee * \\ Department of Electronic Engineering, Seoul National University of Science and Technology, Seoul 01811, Korea; \\ jangsuyeon@seoultech.ac.kr (S.J.); ohhyunwoo@seoultech.ac.kr (H.W.O.); \\ yoonyounghyun@seoultech.ac.kr (Y.H.Y.); hwangdonghyun@seoultech.ac.kr (D.H.H.); \\ jeongwonsik@seoultech.ac.kr (W.S.J.) \\ * Correspondence: seung.lee@seoultech.ac.kr; Tel.: +82-2-970-9021
}

Citation: Jang, S.; Oh, H.W.; Yoon, Y.H.; Hwang, D.H.; Jeong, W.S.; Lee, S.E. A Multi-Core Controller for an Embedded AI System Supporting Parallel Recognition. Micromachines 2021, 12, 852. https://doi.org/ $10.3390 / \mathrm{mi1} 2080852$

Academic Editor: Piero Malcovati

Received: 15 April 2021

Accepted: 15 July 2021

Published: 21 July 2021

Publisher's Note: MDPI stays neutral with regard to jurisdictional claims in published maps and institutional affiliations.

Copyright: (c) 2021 by the authors. Licensee MDPI, Basel, Switzerland. This article is an open access article distributed under the terms and conditions of the Creative Commons Attribution (CC BY) license (https:// creativecommons.org/licenses/by/ $4.0 /)$.

\begin{abstract}
Recent advances in artificial intelligence (AI) technology encourage the adoption of AI systems for various applications. In most deployments, AI-based computing systems adopt the architecture in which the central server processes most of the data. This characteristic makes the system use a high amount of network bandwidth and can cause security issues. In order to overcome these issues, a new AI model called federated learning was presented. Federated learning adopts an architecture in which the clients take care of data training and transmit only the trained result to the central server. As the data training from the client abstracts and reduces the original data, the system operates with reduced network resources and reinforced data security. A system with federated learning supports a variety of client systems. To build an AI system with resource-limited client systems, composing the client system with multiple embedded AI processors is valid. For realizing the system with this architecture, introducing a controller to arbitrate and utilize the AI processors becomes a stringent requirement. In this paper, we propose an embedded AI system for federated learning that can be composed flexibly with the AI core depending on the application. In order to realize the proposed system, we designed a controller for multiple AI cores and implemented it on a field-programmable gate array (FPGA). The operation of the designed controller was verified through image and speech applications, and the performance was verified through a simulator.
\end{abstract}

Keywords: parallel recognition; federated learning; AI processor; embedded system; controller; distributed learning; independent operation

\section{Introduction}

Recent advances in computer and semiconductor process technology have developed artificial intelligence (AI) technology. Due to this trend, adopting AI systems for a variety of applications such as speech recognition [1], medicine [2], and education [3] is widely expanding. Processing large-scale data is one of the basic features of AI technology. As using data centers to process these large-scale data is a widespread technique in AI systems, the demand for data centers is growing because of the increasing expansion of AI systems. Since the amount that the data centers are used is directly tied to the scale of the data, the necessity for systematic exploitation of the data, which dramatically helps to optimize the system to be smart, intelligent, and cost-effective [4], is emerging.

The general machine learning systems adopt the architecture in which the central server processes and trains most of the data and delivers the result to the client [5]. As the clients of this model rarely, if ever, perform the data preprocessing, the workloads of the system are concentrated on the central server. This characteristic makes the system use a high amount of network bandwidth due to sending a huge amount of data, which is rarely preprocessed, from client to server [6]. Moreover, the data communication between the clients and the server on these systems can cause security issues due to sending large amounts of data [7]. Hence, adopting this model to security-sensitive applications 
leads the system to use an enormous amount of additional system resources to grant security. In order to overcome these issues, a new AI model called federated learning was recently presented.

The term federated learning was introduced by McMahan et al. [8]: "We term our approach Federated Learning, since the learning task is solved by a loose federation of participating devices (which we refer to as clients) which are coordinated by a central server". Unlike the previous model, federated learning adopts an architecture in which the clients take care of data training and transmit only the trained result to the central server. McMahan et al. [8] discuss an algorithm for federated learning, and Bonawitz et al. [9] discuss considerations for applying systems and algorithms for federated learning. The federated learning system repeats the training process which is typically driven by model engineers to develop a model for an application [10]. The general training process is as follows [10]:

1. Client selection: The server samples from a set of clients meeting eligibility requirements. For example, mobile phones might only check into the server if they are plugged in, on an unmetered Wi-Fi connection, and idle, in order to avoid impacting the user of the device.

2. Broadcast: The selected clients download the current model weights and a training program from the server.

3. Client computation: Each selected device locally computes an update to the model by executing the training program, which might, for example, run SGD on the local data (as in federated averaging).

4. Aggregation: The server collects an aggregate of the device updates. For efficiency, stragglers might be dropped at this point once a sufficient number of devices have reported results. This stage is also the integration point for many other techniques that will be discussed later, possibly including: a secure aggregation for added privacy, lossy compression of aggregates for communication efficiency, and noise addition and update clipping for differential privacy.

5. Model update: The server locally updates the shared model based on the aggregated update computed from the clients that participated in the current round.

The data training from the client, i.e., client computation, has the effect of encrypting the original data and reducing the amount of data $[11,12]$. This property makes it possible for the system to operate with reduced network resources $[8,13]$ and reinforced data security [14-16]. According to these characteristics, federated learning is being applied to a variety of applications such as smart factories [17], edge device applications [18,19], and end user privacy-sensitive applications.

Figure 1 shows the concept of an AI system with federated learning. Federated learning supports a variety of client systems and is able to perform client computation. Some of these client systems have resource limitations because of various factors. In order to execute client computation with these resource-limited client systems, dealing with these limitations is necessary. One of the applicable methods to solve these limitations is that of composing the client system with multiple embedded AI cores. Although federated learning between heterogeneous devices is the ultimate goal, parallel processing for federated learning among processors within a single device is prioritized, and the flexibility of the system can be provided by adopting this architecture. For the purpose of realizing the system with this architecture, the embedded AI cores need to be utilized and arbitrated by applying an additional controller [20]. In this paper, we propose an embedded AI system for federated learning that can be composed flexibly with AI cores depending on the application. The proposed system requires a specific module to process multiple AI cores in parallel. In order to verify the proposed system, we design a controller for a processor with multiple AI cores. 


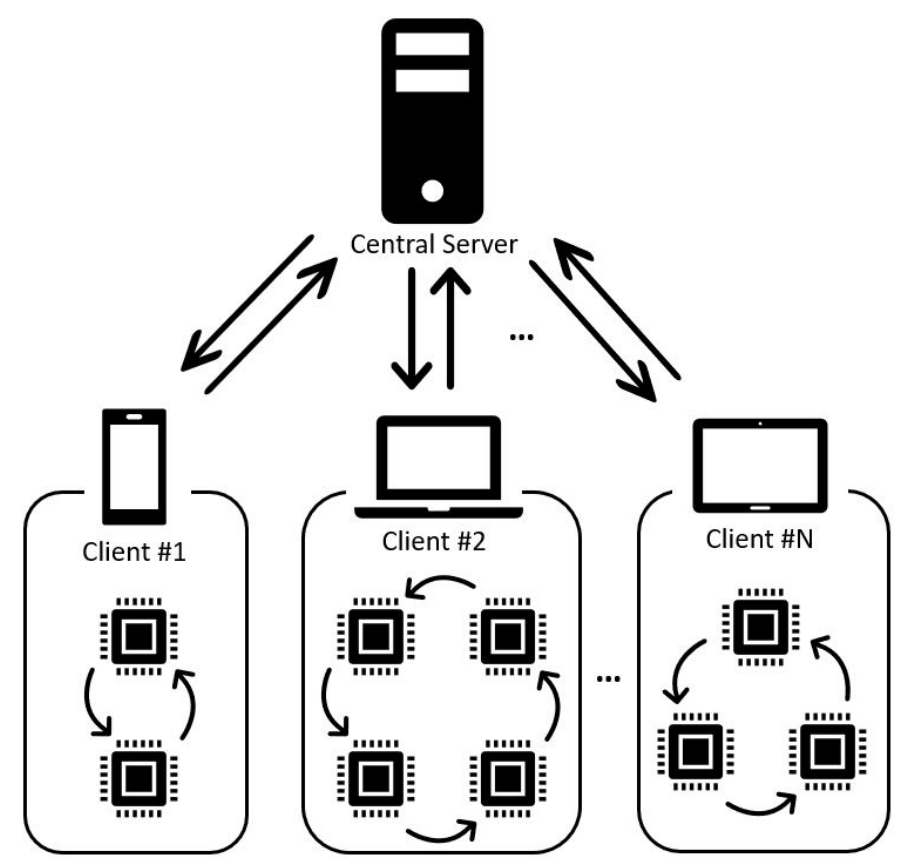

Figure 1. Concept of embedded AI system supporting federated learning.

The designed controller manages the signals for communication with commercialized AI chips and drives the states according to the learning/recognition commands. We validated the feasibility of the proposed system by implementing the controller on a field-programmable gate array (FPGA) and analyzed the performance of the controller by two applications, image recognition and speech recognition. Furthermore, we confirmed the validity of the controller and suitability of the application to the system through performance analysis according to the memory size using a simulator.

The main contribution of this work is as follows:

- Designing and verifying the controller for parallel processing of multiple AI cores.

- Constructing the protocol to transfer the training data and recognition data between the controller and the AI core.

- Performance analysis based on memory usage of the AI system with the $k$-NN algorithm.

As gathering recognition results from AI cores through the proposed controller, it helps to deduce the proper recognition result for the embedded AI system.

The paper consists of the following. Section 2 introduces related works about the AI system and simulator. Section 3 then turns to a detailed description about the AI algorithm and AI system architecture. Section 4 presents the implementation results of the system and analyzes the performance with the results. Section 5 compiles our entire work and draws conclusions.

\section{Related Work}

The recent research related to federated learning is categorized as follows:

- Artificial Intelligence for Embedded Systems;

- $\quad$ Simulating and Performance Analysis for Hardware Implementation.

\subsection{Artificial Intelligence for Embedded Systems}

Due to the limitations of embedded systems, much research in adopting AI in embedded systems concentrates on power and area optimization [21-24]. The research of [21] is one of the cases. The authors of this paper proposed an AI processor for embedded systems that was based on the k-nearest neighbor algorithm and operated by the coupled architecture with a master processor. By adopting the $k$-nearest neighbor algorithm, which 
has relatively small computations compared to other algorithms, the implementation of the AI processor was accomplished with a small area. The AI processor consists of memory cells which store vectorized raw data and distance calculators for classification. The performance and hardware specifications of the AI processor vary with the vector size of the memory cell and the total count of the memory cells. Hence, a framework to reconfigure these properties was built to provide the volatility of the hardware specifications. These properties, such as reconfigurable architecture and coupled architecture, make the AI processor applicable for a variety of applications. The research in [23] presents a dynamic reconfigurable processor (DRP) for embedded AI. The AI processor is composed of an array of processing elements (PE), multiply and accumulate unit (MAC) groups, and direct memory access (DMA). Each MAC group can be equivalent to small tensors or one large tensor in the deep neural network. The MAC supports the half-precision floating-point (FP16) and binary arithmetic mode. Selecting the binary arithmetic mode reduces memory usage but also degrades recognition performance. This architecture provides the flexibility of memory resource usage. In order to verify the DRP, the authors of [23] designed the system with the DRP, ARM CPU, and AXI interface and implemented it on a chip using $28 \mathrm{~nm}$ technology. The evaluation result of the chip shows that the DRP has acceptable performance on arithmetic in deep neural networks.

Unlike the research of $[21,23]$, some studies focus on optimizing the software deep learning algorithm to fit existing embedded system-on-chip (SoC) [25-27]. Adopting the deep learning algorithm with applicable performance to the embedded SoC is extremely hard because of the critically limited memory and storage resources compared to cloud AI or mobile AI devices [25-27]. In order to fix these problems, the authors of $[25,27]$ proposed frameworks for optimized neural network generation. Both frameworks provide quantization of floating-point arithmetic to integer arithmetic and applying memory constants for scaling the neural network for each device. The framework of [28] has two fundamental features. One is a neural architecture search (NAS). By providing an NAS that works with a memory size constraint, the neural architecture which is suitable to the microcontroller can be searched [25]. The other is a memory-efficient inference library. In contrast to traditional inference libraries, which depend on a runtime interpreter, the library adopts pre-runtime compilation to operate the system. This characteristic leads to significantly reducing the memory and computing overhead caused by the management of the metadata of variables. As a result, adopting the framework results in a remarkable reduction in latency and maximum SRAM usage. The framework in [27] concentrates on software optimization, which can be achieved by reflecting the configuration of mirror layered memory architecture and DMA provided by the target hardware. The result shows that the memory transferring overhead caused by cache memory is almost hidden. These studies suggest the possibilities of applying AI to the embedded SoCs.

\subsection{Simulating and Performance Analysis for Hardware Implementation}

When applying either AI or other features in embedded systems, searching and selecting hardware specifications that meet the performance requirements of each application are as important as designing the embedded system. Hence, many studies have been performed to build a framework to simulate and design the system efficiently [21,28-30]. The research of [29] is a suitable example of this research category. This paper presents a simulator to analyze the performance of the embedded AI to help to decide on the specifications of the embedded AI, such as the AI processor described in Section 2.1. The simulator can be executed with the sample dataset which is used for certain applications to secure the reliability of the results. As the specifications of the AI processor are related to memory size and the number of categories, the simulator provides the configuration of these options. As analyzing the performance after finishing the implementation of hardware is a far more complex process than analyzing with simulations, using the simulator to decide the attributes of the embedded AI before implementing the hardware increases the efficiency of the design and verification process of the system. 
Many studies have been conducted that can be applied to federated learning as explained above. The main contribution of this work is as follows: to design and verify the controller of the embedded AI processors that is applicable to federated learning systems using the methodology of the above studies.

\section{System Architecture}

The embedded AI system performs learning and reasoning by using various datasets such as image or speech data. The embedded AI system allows the AI core selected by the user to operate AI functions (e.g., learning or recognition operations) through parallelized multiple AI cores. The AI core is a General Vision pattern recognition chip that performs recognition through the $k$-NN algorithm. In order to perform pattern recognition, the user should choose a method among the recognition stage and I2C slave controller and a method of directly controlling the neuron register.

In the proposed system, for the daisy-chain connection of several AI cores and optimization of the controller, the neuron register is directly accessed to control the neuro cell memory. For the purpose of utilizing the parallelized multiple AI cores efficiently, an AI processor needs a controller for multiple AI cores. Additionally, an interface is required to communicate data. The appropriate protocol for the AI core is used for the system to facilitate the repetitive AI operation. The algorithm and microarchitecture of the embedded AI system with operation flow are described below.

\section{1. k-NN Algorithm}

The $k$-NN algorithm is a distance-based machine learning algorithm used for classifying the category of the data. The basic concept of the $k-\mathrm{NN}$ algorithm is that of finding the closest values between the input data and the trained dataset. The constant $\mathrm{k}$ in $k$-NN indicates how many values the algorithm will find. A distance calculation method between the trained data and input vector varies. In general, Euclidean distance or Manhattan distance is used for distance calculation. To classify the data with maintaining the concept of the $k$-NN algorithm, the information of the trained data and the input data, which contains a variety of characteristics, needs to be expressed to a group of numerical values and a single category of information. Thus, the data which is used for the algorithm is expressed as a group of n-dimensional vectors and the category. Figure 2 presents the mechanism of the $k$-NN algorithm when the data are expressed as a two-dimensional vector. The algorithm operates by two key features. One is training the system with the algorithm by storing the vector data with category information. The other is a classification of the input data.

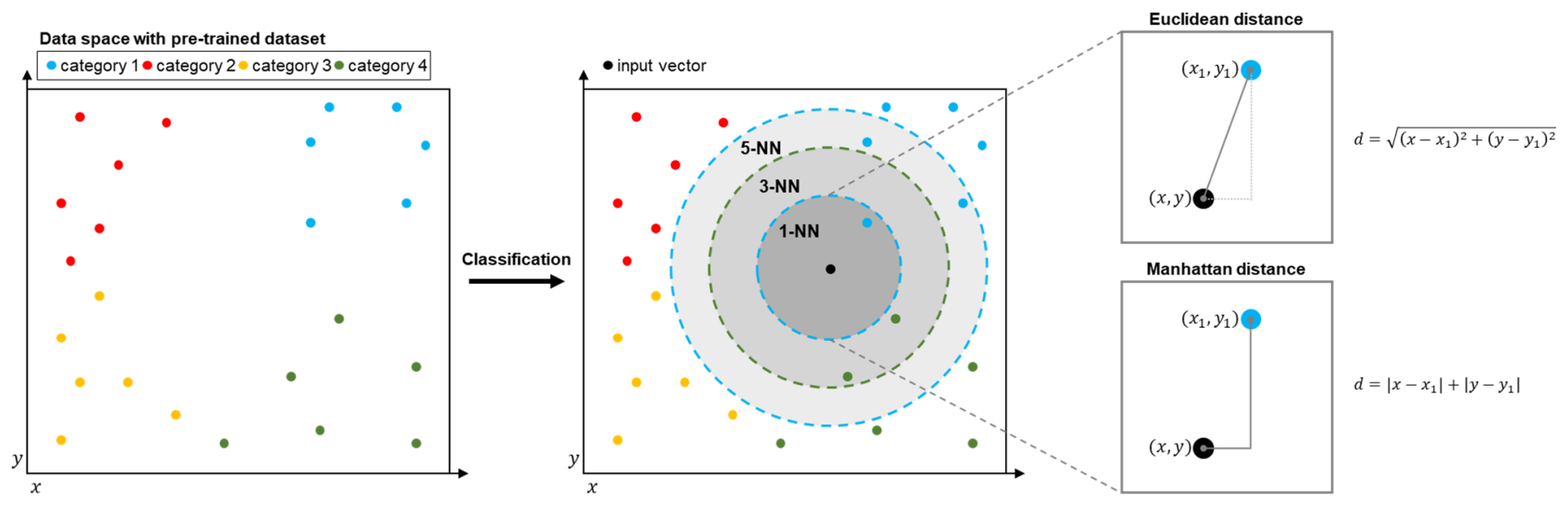

Figure 2. Visualization of the k-nearest neighbor algorithm.

Algorithm 1 presents the classification process. During the classification, the $k$-NN algorithm finds a $k$ number of data with the shortest distance in the trained dataset. After finding the nearest data, the algorithm extracts the most frequent category from a number 
of nearest data. Due to the architecture of these key features, the most frequent category can be more than one when the $k$ value is more than one. To avoid this problem, an additional method to give proper weight to the found data or setting the $k$ value to one is required. As shown in Algorithm 1, an increase in the constant $k$ leads the complexity and iteration count of the algorithm to be higher. This attribute is not appropriate for the embedded system which is sensitive to area usage and power consumption. Therefore, we set the $k$ value to one to minimize the area usage and power consumption of the system. Algorithm 2 shows the minimized algorithm by setting the constant $k$ to one.

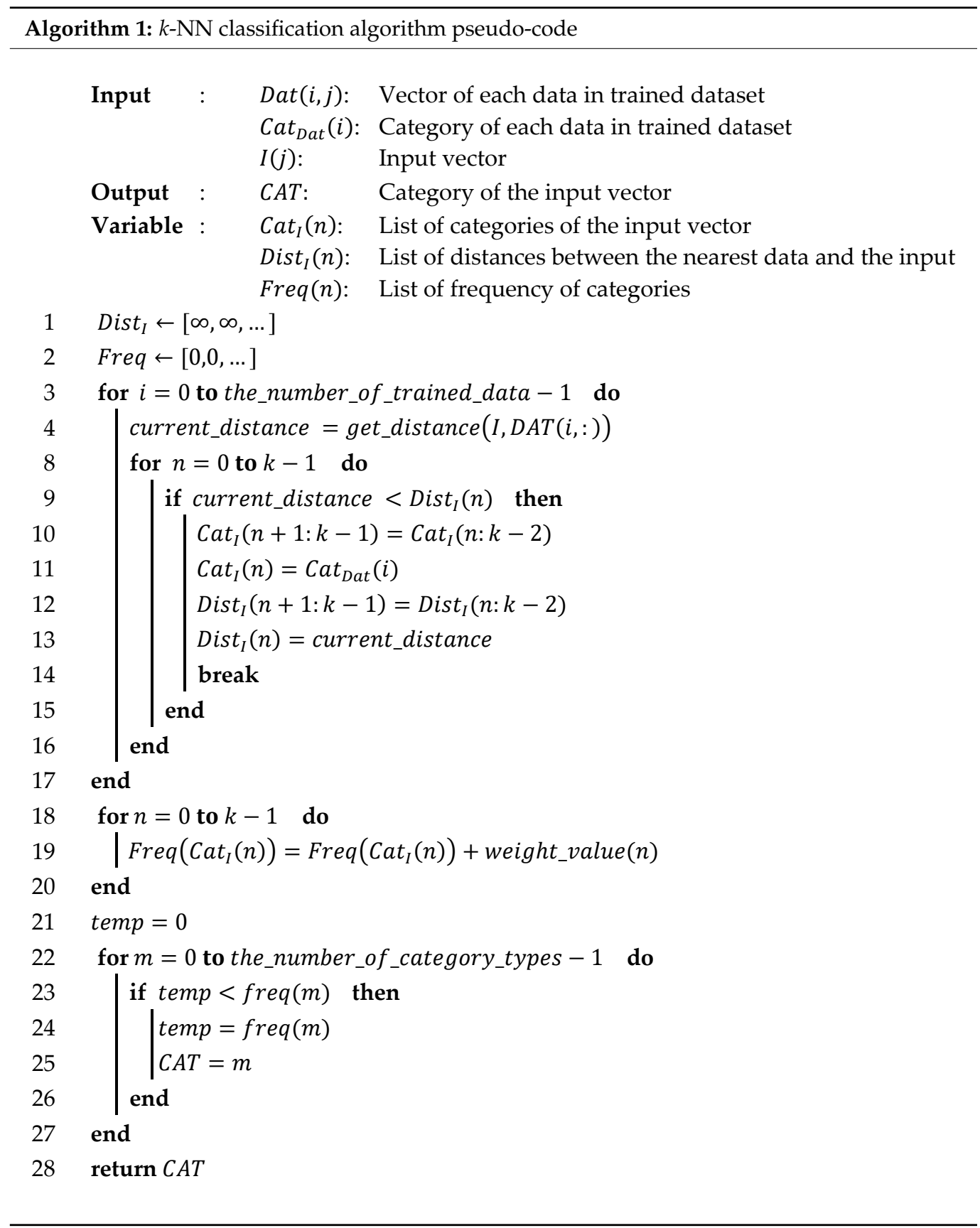




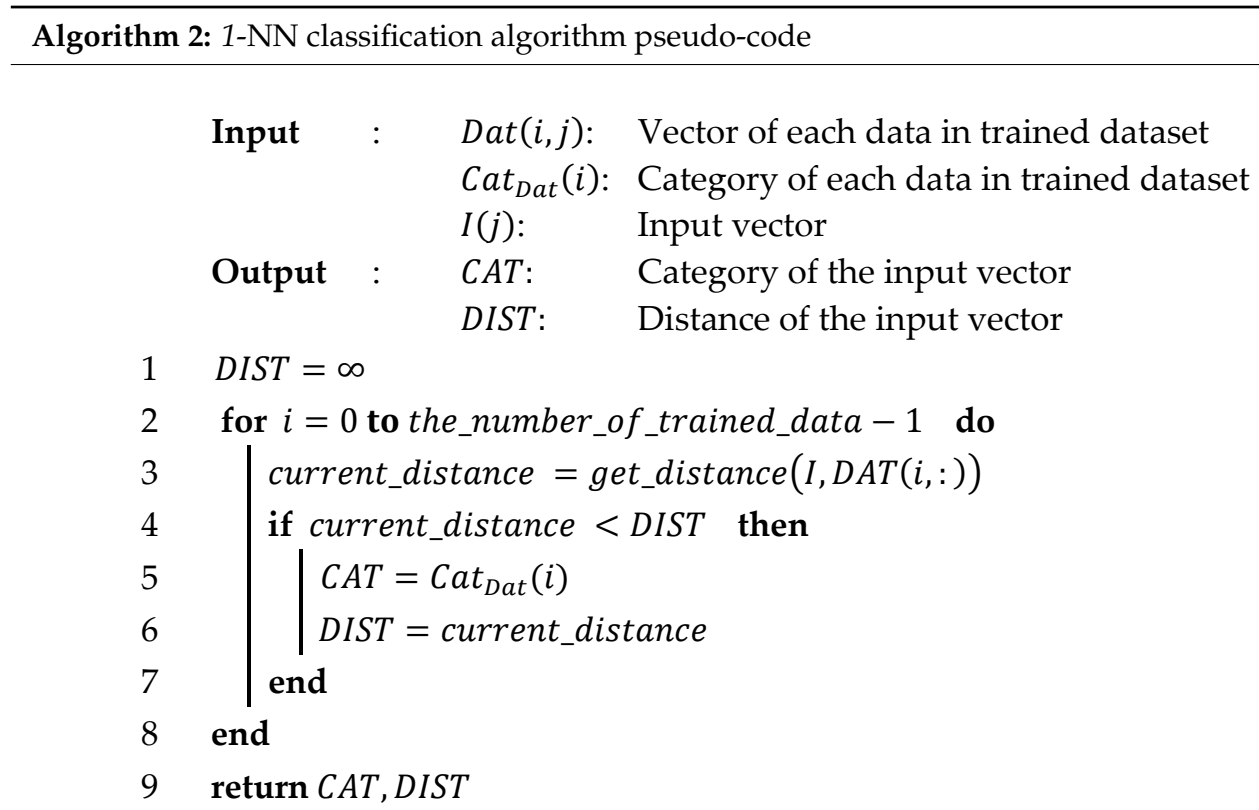

The $A I$ core performs key features of the $k-\mathrm{NN}$ algorithm except extracting from the most frequent category. It provides the category and distance values sequentially in order of the shortest distance between the input vector and the data from the trained dataset. The specifications, such as the constant $k$ and number of dimensions, can be configured by an external device. Additionally, the AI core applied the Manhattan distance method to lower the complexity of calculation in order to operate with low power consumption. The $A I$ core can have 1024-byte vectors that are composed of up to 256 components of 8-bit value, and vectors can include 15-bit of category information [31]. Each datum in the vector is expressed as a 1-byte unsigned integer.

\subsection{Microarchitecture}

Figure 3 illustrates the block diagram of the embedded AI system. The embedded AI system includes an interface for communication with other systems through an external data transceiver and an AI processor with multiple AI cores for learning/recognition operations.

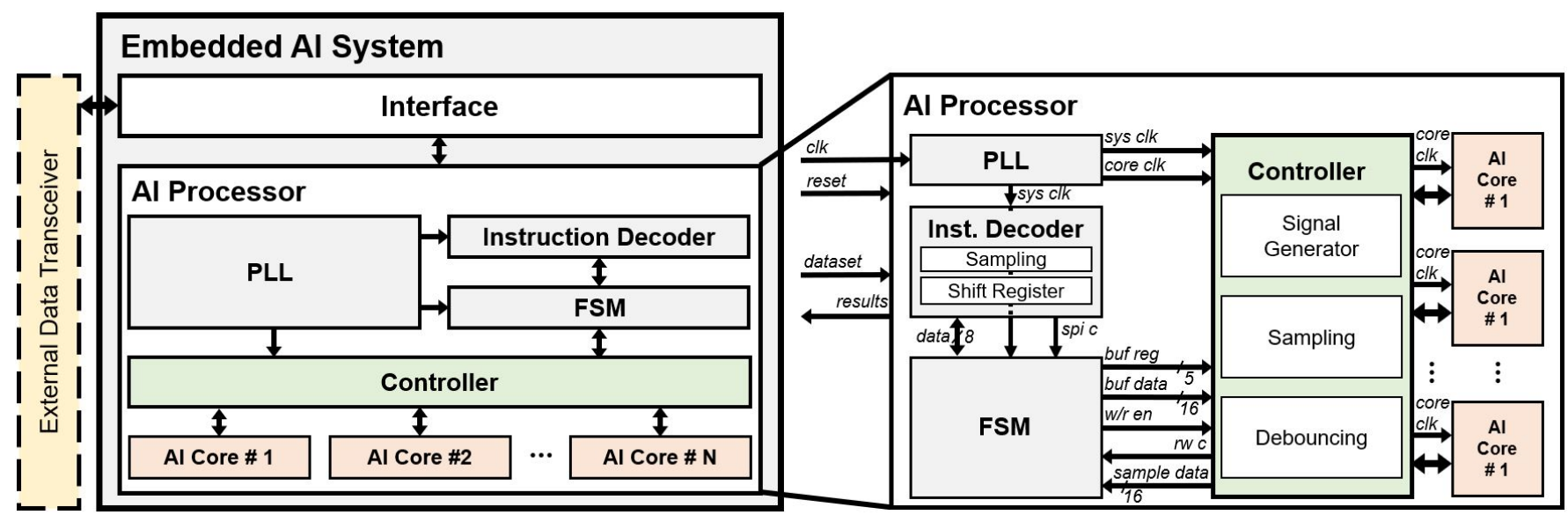

Figure 3. Block diagram of embedded AI system and AI processor in detail.

The external data transceiver sends a certain dataset for learning and recognizing operations, which is shaped to a protocol based on a serial peripheral interface (SPI) protocol, to the interface. The AI processor includes the phase locked loop (PLL), instruction 
decoder, finite state machine (FSM), controller for efficient management of AI cores, and AI cores. The PLL generates a $64 \mathrm{MHz}$ system clock for the controller and a $16 \mathrm{MHz}$ core clock for the AI cores. The instruction decoder in the AI processor receives the dataset through the interface by decoding a protocol for learning/recognition.

Figure 4 shows the protocol for learning and recognition. The protocol basically contains a 1-byte setup value composed of configurations such as read/write select $(R W$ $S E L)$, write mode, and register address (REG). RW SEL is required to distinguish between learning and recognition operations by the FSM. After the division operation, the system decides whether it is single-mode or not using write mode. In the case of learning in singlemode, the setup value to configure the interface to single write mode and set the destination to a certain address is first sent to the interface. Next, two bytes of the learning data are sent. In the case of learning in sequential mode, the setup value to configure the interface to sequential write mode and set the destination to a certain address is first sent to the interface. After sending the setup value, the 2-byte length value of learning data and a number of learning data are sent sequentially. When an AI core operates learning or recognition, a 5-bit register address, data, and category value are transmitted via control lines [31] through the FSM and the controller. Due to the protocol, the FSM and the controller generate control signals and send data for $A I$ cores without storing the dataset.

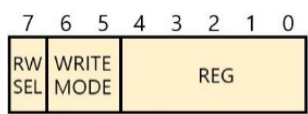

setup value 1-byte

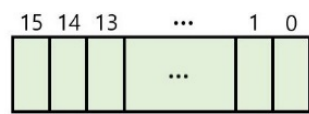

data length 2-byte

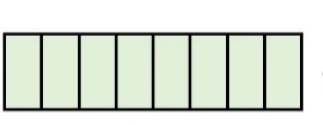

data

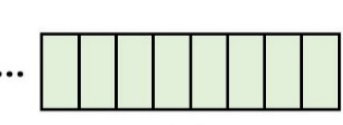

data

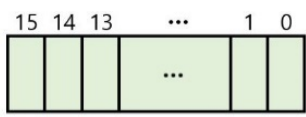

category 2-byte

Figure 4. Protocol for learning and recognition in detail.

The FSM is a module to provide well-ordered communication between the instruction decoder and the controller. Figure 5 shows the FSM in detail, which operates according to the active signal from the instruction decoder. The FSM performs learning and recognition operations based on the serial interface and distinguishes between learning and recognition operations. Furthermore, the FSM can discriminate between single and sequential modes. In case of the single-mode, the FSM only needs 2-byte data to receive read data or transmit write data. In the other case, the FSM requires 1-byte data for data length and continuous data including category data. When the result data from the AI core are received through the controller, the result data are sent to the instruction decoder. In order to perform the learning/recognition process or communication when the FSM transmits data to the controller or instruction decoder, it receives a flag signal from each module to prevent data collision. In addition, the FSM generates different signals according to the learning/recognition process so that the user can control the neuron register of the AI core. The architecture of the FSM allows the AI processor to perform continuous learning and recognition operations.

The controller is connected in parallel with the multiple AI cores, and each AI core can be operated independently through the controller. The controller generates control signals that can directly control the operation of the AI core [31] and transmits it along with the data. In the case of the learning process, the controller transmits the data to the selected AI core when it receives the buffer data and the write enable signal from the FSM. In the case of the recognition process, the controller transmits the data and read enable signal to the selected AI core. After that, the controller receives a result of the distance calculation and the category value from the selected AI core and transmits them to the FSM. When the controller receives the result data from the AI cores, the sampling block is performed to receive data reliably. 


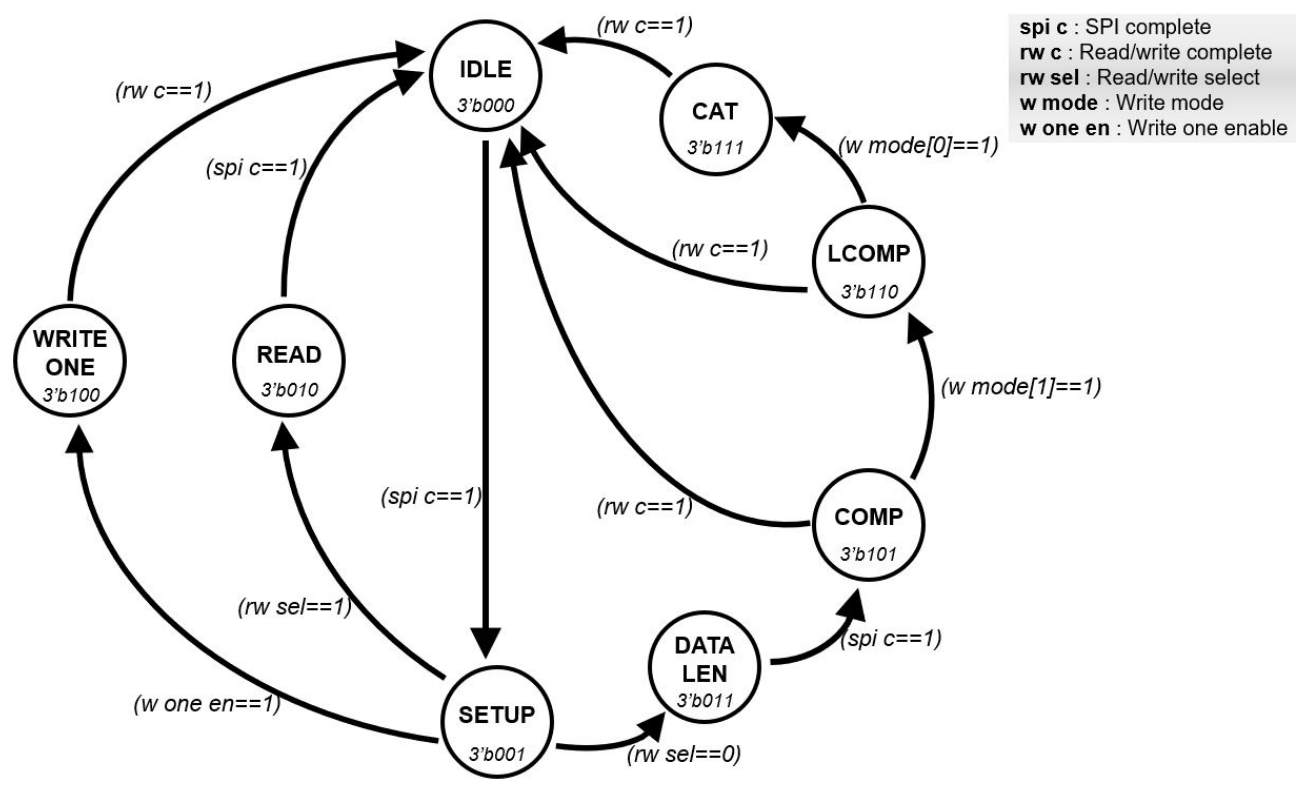

Figure 5. The architecture of the FSM.

The AI core needs to receive a dataset (vector) before performing learning or recognition. The method of transmitting the vector to the AI core is called vector broadcasting [31]. In vector broadcasting, the processes called the component (COMP), which stores 1-byte data, and the last component (LCOMP), which is performed after COMP is finished, exist. The COMP can be performed up to 255 times. In the case of learning, the state called the CAT, which stores the category data, is performed after the vector broadcasting is finished. After the learning operation, the AI core that receives the test vector calculates the distance between learned and test data. The AI core reads the category value from the recognition result of the READ state, which has the lowest distance value.

\section{Implementation and Analysis}

In order to evaluate the embedded AI system, we implemented an AI processor which includes multiple AI cores. Figure 6 shows the hardware experiment environment in which the controller and multiple AI cores are connected in parallel. We implemented the controller on an Intel FPGA (Cyclone III) and used General Vision's pattern recognition chip (CM1K) for the AI core. Using the verified AI core helps to clarify the functional verification of the designed controller. Previous to testing the implemented processor, we simulated the processor using a simulator written in Python that has the same capability as the AI core for performance analysis. Before applying the application to the embedded AI system, the simulator that mimics the structure of the AI core can be used to check the suitability of the application. We analyzed the performance according to the memory size of the virtual AI core and performed functional verification of the system through image and speech applications.

\subsection{Implementation}

\subsubsection{Case Study 1: Image Recognition}

The MNIST dataset of handwritten digits has a training set of 60,000 examples and a test set of 10,000 examples. The digits are in a grayscale image, size-normalized, and centered in a fixed-size image [32]. In order to uniformly learn the AI core by category, data are evenly sampled from the MNIST dataset. The sampled training data are used for each AI core to learn. 


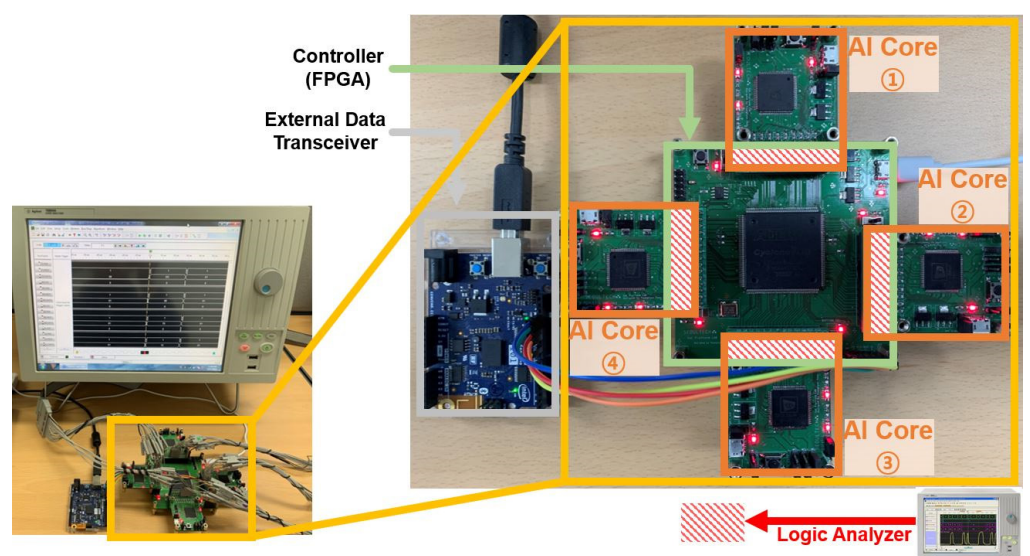

Figure 6. Experimental environment of the FPGA implementation.

With the purpose of verifying the functionality of the designed controller in the image application and the parallel processing of multiple AI cores, we constructed the environment of the implementation. Figure 7 shows the result of measuring the signals through the logic analyzer for the distance result value and the category value of each AI core. The recognition results from each AI core are collected to the controller. Each AI core learned the same category (from 0 to 3 ) through different training data and received the same test data for recognition.

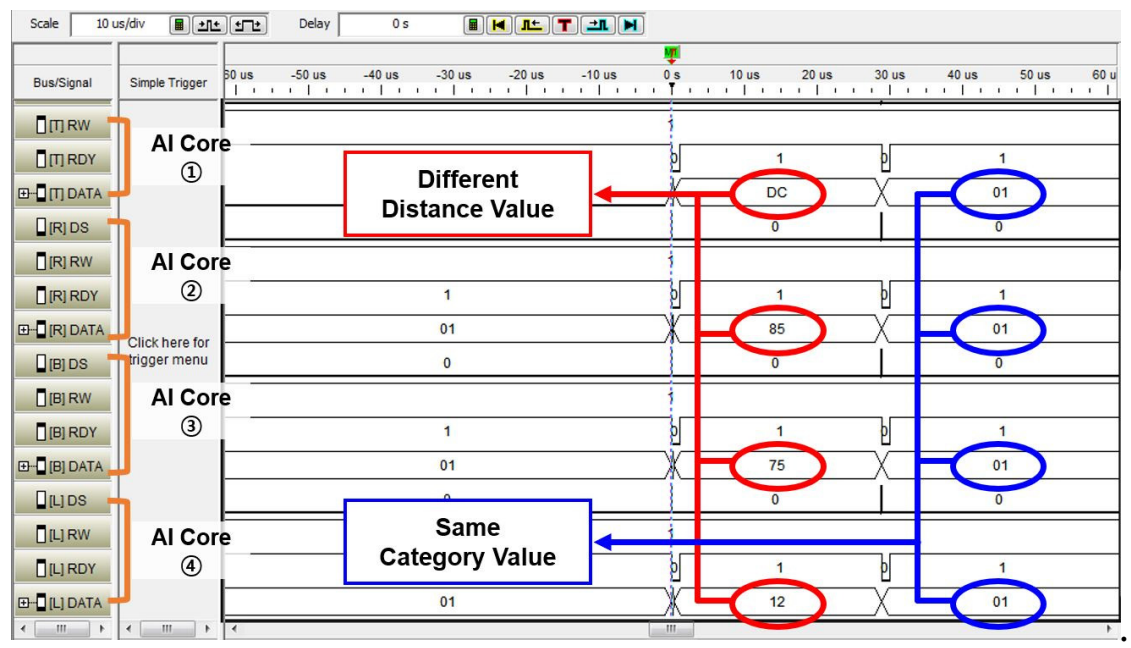

Figure 7. Image recognition results on FPGA implementation.

Each $A I$ core outputted a result of distance calculation between the trained data and test data through the $k$-NN classifier in the AI core and a category value accordingly. As a result of the recognition, it was confirmed that the result values of the distances $0 \times \mathrm{DC}$, $0 \times 85,0 \times 75$, and $0 \times 12$ are sequentially from the first $A I$ core, and the category values of all $A I$ cores are $0 \times 1$, indicating the same value. The distance calculation results from the AI cores were obtained by the $k-\mathrm{NN}$ algorithm in Section 3, and each AI core evaluates a category value based on the distance calculation result. This meant that the trained data of AI core (1) was furthest from the test data, and the trained data of AI core (4) was most similar to the test data. The AI cores were operated independently by the controller as each AI core outputted a difference distance value. As a result, the controller gathers the different distance values and same category values from each AI core. 


\subsubsection{Case Study 2: Speech Recognition}

As speech technology advances, research of speech recognition is increasing, but the availability of datasets has not widened [33]. In order to train and evaluate the embedded AI system for a speech application, the speech command built in Google Brain [33] is used as follows:

- Google Brain's 2 categories Speech Command;

- Google Brain's 3 categories Speech Command;

- Two categories of Recorded Voice Data.

In this experiment, the above speech commands were selected in consideration of the embedded AI system. Each speech command is evenly sampled to train the AI core by category. The sampled data is transformed into a two-dimensional (2D) signal through the short-time Fourier transform (STFT). The 2D signal is resized to be used as an input to the AI core and converted into a 1D signal. Finally, the converted data is used in the embedded AI system. In order to verify the functionality of the controller in the speech application, the experiment was implemented as in Case 1 (image application). Each AI core learned Case 2-3 (two categories of recorded voice data) training data recorded in different voices and recognized the test data. As the result, the controller for multiple AI cores operated successfully in the speech application.

\subsection{Analysis}

Table 1 shows the power consumption and logic element usage of the controller on the Intel EP3C25Q240C8N FPGA and the power consumption of the AI core, which is the General Vision chip [31]. The synthesis of the controller was executed with Quartus II 13.1 version software. The power consumption of the controller was obtained with PowerPlay Power Analyzer in Quartus II 13.1 version. The result presents that the proposed system is suitable for an embedded device.

Table 1. Resource utilization of the AI processor.

\begin{tabular}{|c|c|c|c|}
\hline & & & Synthesis \\
\hline \multirow{3}{*}{$\begin{array}{c}\text { Controller } \\
\text { (Cyclone III: EP3C35Q240C8N) }\end{array}$} & \multicolumn{2}{|c|}{ Total logic elements ${ }^{1}$} & $182(<1 \%)$ \\
\hline & \multicolumn{2}{|c|}{$\begin{array}{c}\text { Core dynamic } \\
\text { power consumption }\end{array}$} & $1.62 \mathrm{~mW}$ \\
\hline & \multicolumn{2}{|c|}{$\begin{array}{c}\text { Core static } \\
\text { power consumption }\end{array}$} & $81.03 \mathrm{~mW}$ \\
\hline \multirow{3}{*}{$\begin{array}{l}\text { AI Core } \\
\left(\mathrm{CM} 1 \mathrm{~K}^{2}\right)\end{array}$} & \multirow{2}{*}{ Single chip } & Idle mode & $15 \mathrm{~mW}$ \\
\hline & & Active mode & $\sim 275 \mathrm{~mW}$ \\
\hline & Multiple chip & - & $\sim 500 \mathrm{~mW}$ \\
\hline
\end{tabular}

${ }^{1}$ Total of 24,$624 ;{ }^{2}$ referenced General Vision document.

The performance was verified by assuming a virtual AI core with a memory size of $16 \mathrm{~KB}$ and $64 \mathrm{~KB}$ through a simulator. The experiment was conducted for each case above, and learning was conducted using only the training data of each dataset. In order to evaluate the performance of each case, the value of accuracy and recall, one of the performance indicators of the AI algorithm, was measured. In addition, we used datasets for learning and evaluation for performance evaluation. Case 1 (image application) trained 10 categories of the MNIST dataset, and Case 2-1 (speech application) trained two categories of speech command data from Google Brain. Figure 8 shows the performance according to the data size (vector size) and the number of neurons (\# of neurons) in the AI core. 


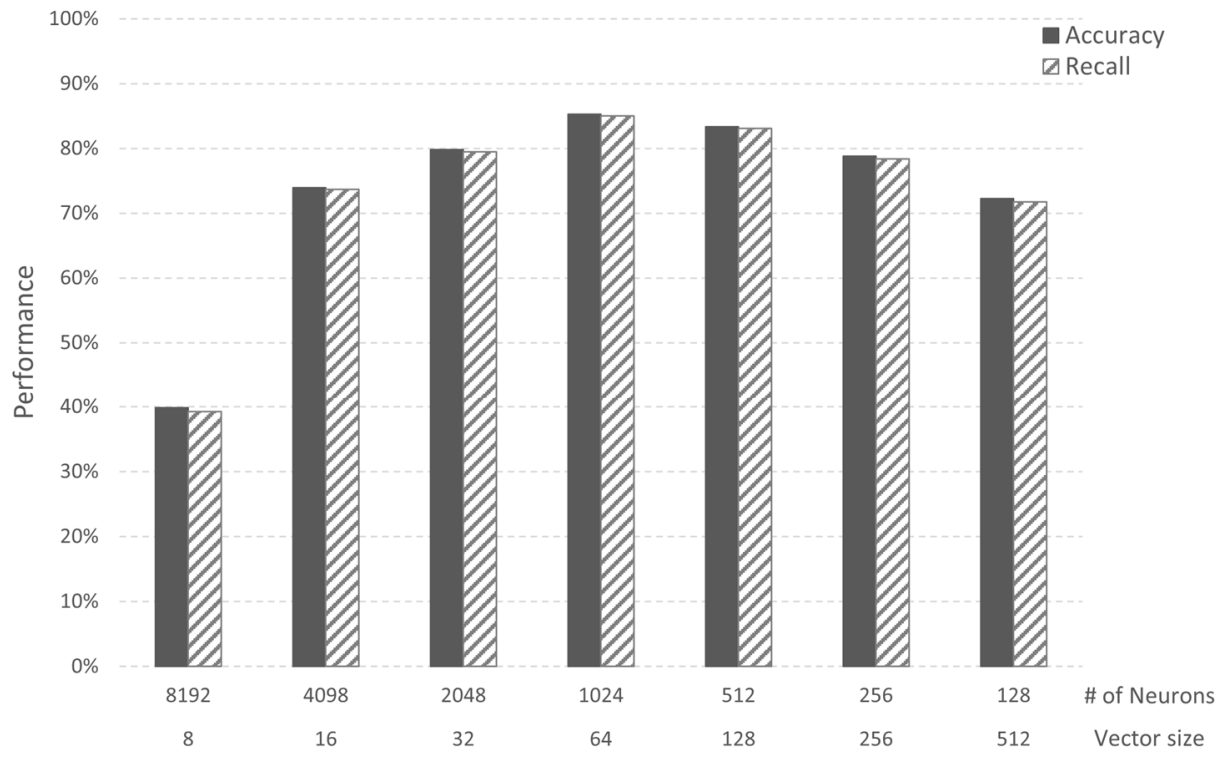

(a)

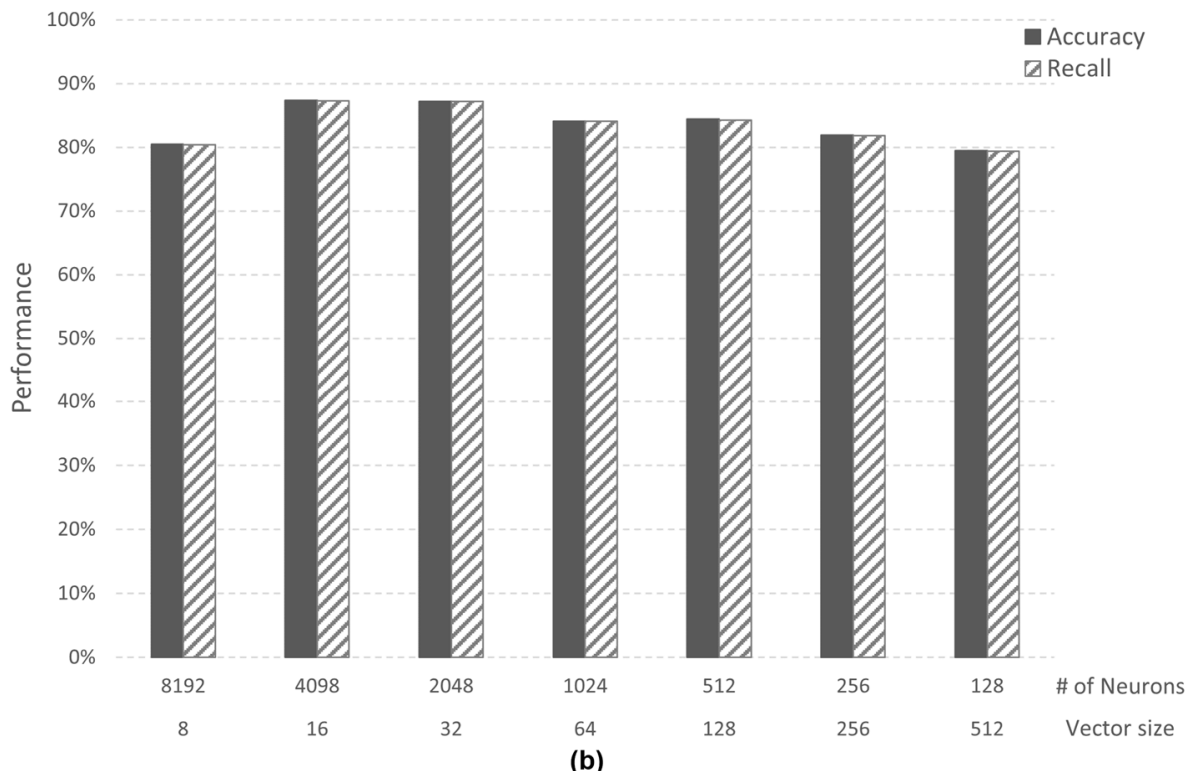

Figure 8. (a) Case 1 performance of recognition; (b) Case 2-1 performance of recognition.

In Case 1, when the vector size is 64 bytes and the number of neurons is 1024 , the $A I$ core has both an accuracy and recall of $85 \%$. In Case $2-1$, when the vector size is 16 bytes and the number of neurons is 4098 , the $A I$ core has both an accuracy and recall of $87 \%$. However, since the AI core experimented with has a maximum number of neurons of 1024, the maximum performance in Case $2-1$ is $84 \%$. Through this analysis, we confirmed that the minimum vector size required in both Cases is 64 bytes. Case 2-2 (speech application) trained three categories of speech command data from Google Brain. In Case 2-2, when the vector size is 64 bytes and the number of neurons is 1024, both accuracy and recall are $73 \%$. Case 2-2 is confirmed to have lower performance than Case 2-1 in the same simulator environment because there are more categories than Case 2-1. Figure 9 shows the accuracy of Case 1 and Case 2-2. When the vector size is 8, Case 2-2 shows higher performance than Case 1 . However, when the vector size is 16 or higher, Case 1 shows better performance than Case 2-2. 


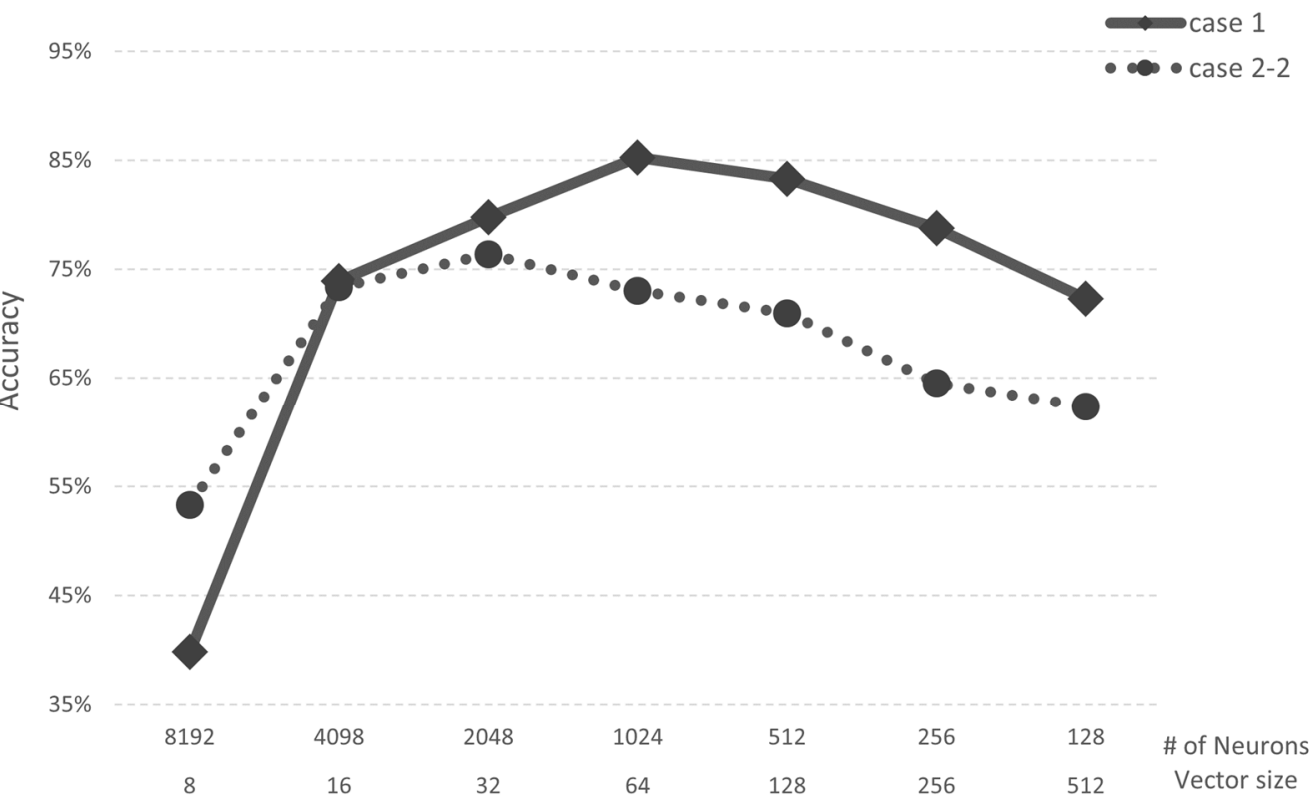

Figure 9. Accuracy of Case 1 and Case 2-2.

Figure 10 shows accuracy analysis according to $16 \mathrm{~KB}$ and $64 \mathrm{~KB}$ memory size of the AI core in Case 1 and Case 2-2. The number of neurons (\# of neurons) changes according to the memory size since the vector size is fixed. The accuracy of $64 \mathrm{~KB}$ memory shows higher accuracy than $16 \mathrm{~KB}$ memory in both cases. In Case 1, the accuracy of $64 \mathrm{~KB}$ memory is up to $17.87 \% \mathrm{p}$ (percentage point) higher than $16 \mathrm{~KB}$ memory, and Case 2 is $10.09 \%$ p higher.

Through the experiment, the suitability of the application could be verified before being applied to the hardware, and the validity of the controller was verified through performance analysis according to the memory size. In addition, it was confirmed that the controller of the proposed system operates successfully, and the parallel processing functionality in the embedded AI system was determined.

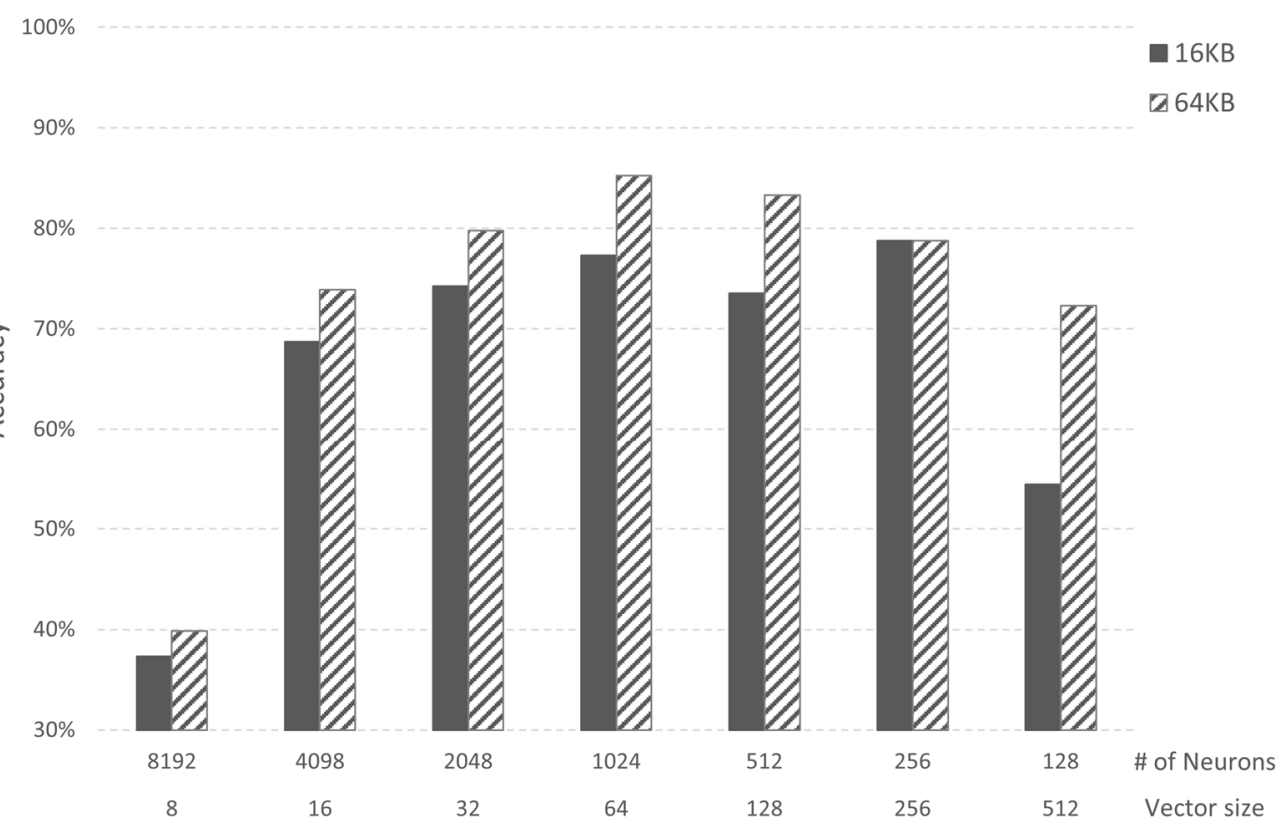

(a)

Figure 10. Cont. 


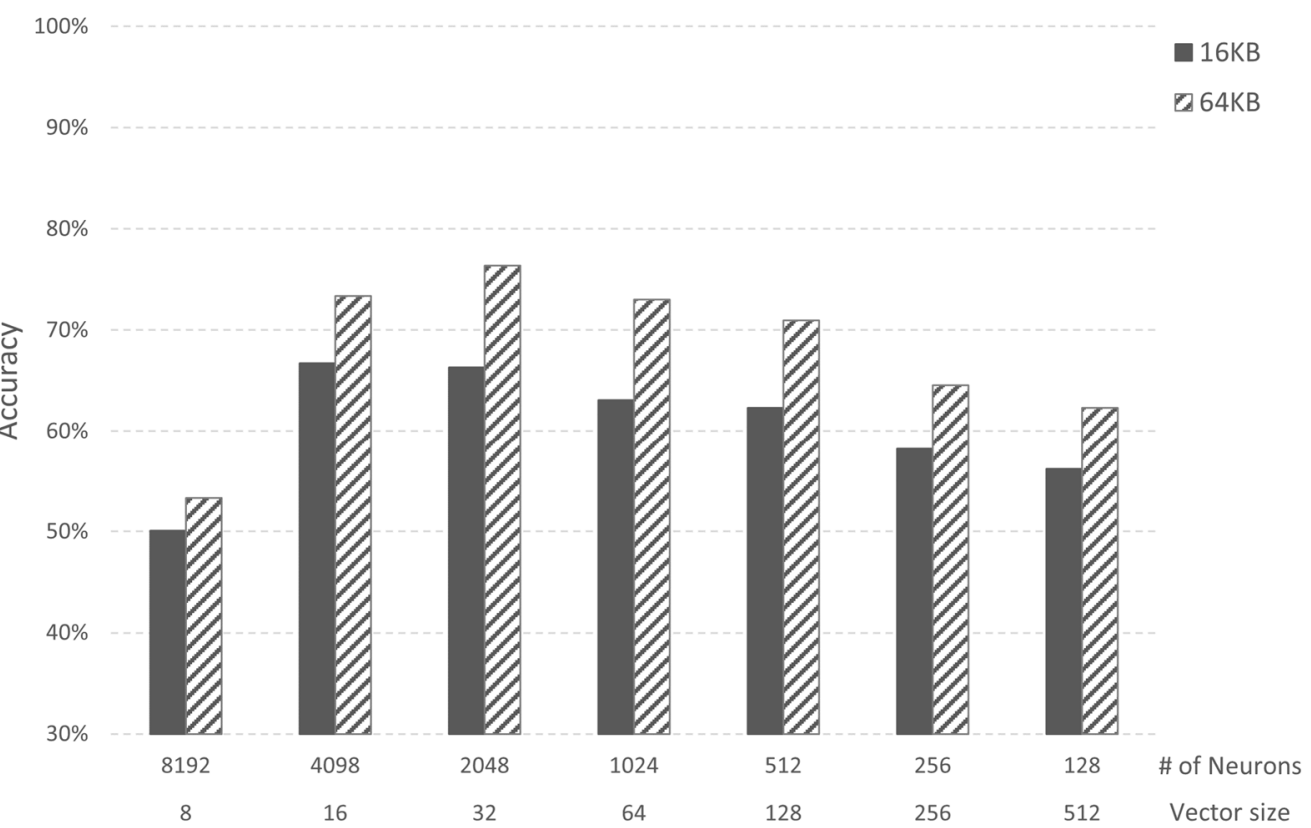

(b)

Figure 10. (a) Accuracy comparison in Case 1; (b) Accuracy comparison in Case 2-2.

Since we used the $k$-NN algorithm, the time it takes to learn and infer data, except preprocessing, is largely independent of the class of data. We measured the time taken for learning and inference using the MNIST dataset, and the results are shown in Figure 11. We used the simulator to measure the time, and the system specifications were an Intel i9-11900K (Intel Corporation, Santa Clara, CA, USA), 94.2 GB Memory, and an Nvidia RTX-3090 (Nvidia Corporation, Santa Clara, CA, USA). When the number of neurons is 8192 , the training time is $1.071 \mathrm{~s}$. As the vector size decreases, the time required for learning decreases dramatically: $0.278 \mathrm{~s}$ when there are 4098 neurons. Thus, the case of 1024 neurons shows the highest performance: it takes only $0.017 \mathrm{~s}$ for learning and $0.003 \mathrm{~s}$ for recognition. The recognition times show less than $0.03 \mathrm{~s}$ regardless of the number of neurons.

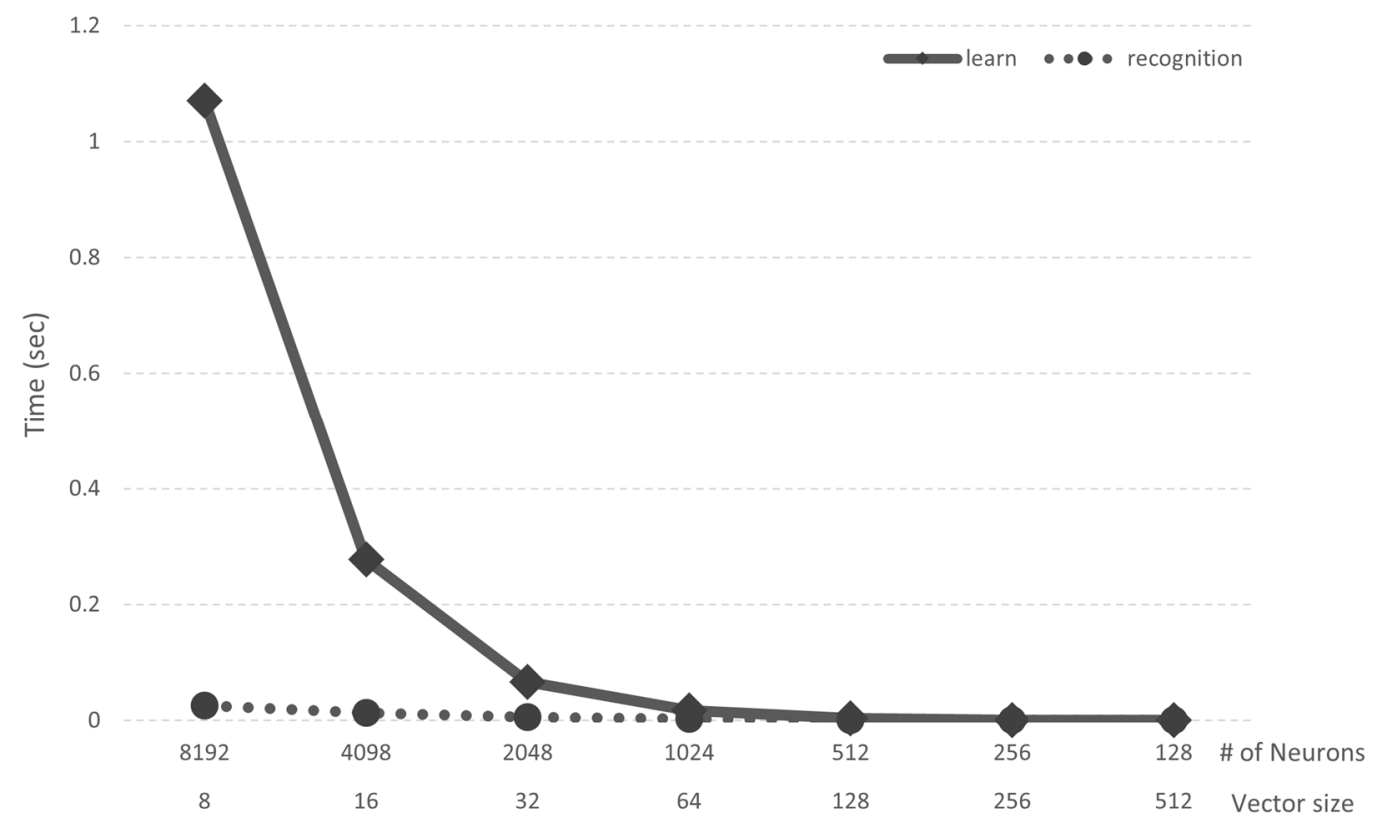

Figure 11. Time consumption of training and recognition. 


\section{Conclusions}

Federated learning enables distributed clients to learn prediction models while collecting training data on the clients. For this, an embedded system that supports federated learning is required. This paper proposed a controller for parallel recognition among AI cores within an embedded AI system. The AI processor in the embedded AI system contains several AI cores in parallel and a controller for efficient operation of the AI cores. The controller independently operated multiple AI cores and flexibly configured the number of AI cores according to the requirements of the application to be applied. In addition, the performance and limitations were determined in advance through virtual artificial intelligence cores and simulators. In order to verify the functionality of the designed controller, image and voice recognition were performed in the FPGA, and the performance according to the number of neurons and data size in the AI core was confirmed through the simulator. According to hardware design and the analysis, it is possible to confirm the potential of the embedded AI system in which multiple artificial intelligence cores can be united. Furthermore, the embedded AI system can choose the proper recognition result from gathered recognition results by the controller through polling or a comparison among collected results.

In future work, we will apply our controller for an embedded intelligence processor to various embedded devices and experiment with the association between heterogeneous embedded systems.

Author Contributions: Conceptualization, S.J. and Y.H.Y.; methodology, Y.H.Y.; data curation, D.H.H.; investigation, S.J., H.W.O., and W.S.J.; writing—original draft preparation, writing-review and editing, S.J. and S.E.L.; supervision, S.E.L.; All authors have read and agreed to the published version of the manuscript.

Funding: This research was funded by the National Research Foundation of Korea (NRF) grant funded by the Korean government (MSIT); No. 2019R1F1A1060044, 'Multi-core Hardware Accelerator for High-Performance Computing (HPC)'.

Conflicts of Interest: The authors declare no conflict of interest.

\section{References}

1. Liu, C.; Wang, L.; Dang, J. Masking based Spectral Feature Enhancement for Robust Automatic Speech Recognition. In Proceedings of the IEEE International Conference on Artificial Intelligence and Computer Applications (ICAICA), Dalian, China, 27-29 June 2020; pp. 287-291.

2. Jacob, S.; Menon, V.G.; Al-Turjman, F.; Vinoj, P.G.; Mostarda, L. Artificial Muscle Intelligence System with Deep Learning for Post-Stroke Assistance and Rehabilitation. IEEE Access 2019, 7, 133463-133473. [CrossRef]

3. Chen, L.; Chen, P.; Lin, Z. Artificial Intelligence in Education: A Review. IEEE Access 2020, 8, 75264-75278. [CrossRef]

4. Kibria, M.G.; Nauyen, K.; Villardi, G.P.; Zhao, O.; Ishizu, K.; Kojima, F. Big Data Analytics, Machine Learning, and Artificial Intelligence in Next-Generation Wireless Networks. IEEE Access 2018, 6, 32328-32338. [CrossRef]

5. Kuflik, T.; Kay, J.; Kummerfeld, B. Challenges and Solutions of Ubiquitous User Modeling. Ubiquitous Display Environments; Krüger, A., Kuflik, T., Eds.; Springer: Berlin/Heidelberg, Germany, 2012; pp. 7-30.

6. Yang, T. Trading computation for communication: Distributed stochastic dual coordinate ascent. In Proceedings of the 26th International Conference on Neural Information Processing Systems, Lake Tahoe, NV, USA, 5-10 December 2013; Curran Associates: Red Hook, NY, USA; Volume 1.

7. Li, T.; Sahu, A.K.; Talwalkar, A.; Smith, V. Federated Learning: Challenges, Methods, and Future Directions. IEEE Signal Process. Mag. 2020, 37, 50-60. [CrossRef]

8. McMahan, H.B.; Moore, E.; Ramage, D.; Hampson, S.; y Arcas, B.A. Communication-Efficient Learning of Deep Networks from Decentralized Data. In Proceedings of the 20th International Conference on Artificial Intelligence and Statistics, Fort Lauderdale, FL, USA, 20-22 April 2017.

9. Bonawitz, K.; Eichner, H.; Grieskamp, W.; Huba, D.; Ingerman, A.; Ivanov, V.; Kiddon, C.; Konečný, J.; Mazzocchi, S.; McMahan, H.B.; et al. Towards Federated Learning at Scale: System Design. arXiv 2019, arXiv:1902.01046.

10. Kairouz, P.; McMahan, H.B.; Avent, B.; Bellet, A.; Bennis, M.; Bhagoji, A.N.; Bonawitz, K.; Charles, Z.; Cormode, G.; Cummings, R.; et al. Advances and Open Problems in Federated Learning. arXiv 2021, arXiv:1912.04977.

11. Kim, H.; Park, J.; Bennis, M.; Kim, S. Blockchained On-Device Federated Learning. IEEE Commun. Lett. 2020, $24,1279-1283$. [CrossRef] 
12. Tran, N.H.; Bao, W.; Zomaya, A.; Nguyen, M.N.H.; Hong, C.S. Federated Learning over Wireless Networks: Optimization Model Design and Analysis. In Proceedings of the IEEE Conference on Computer Communications, Paris, France, 29 April-2 May 2019; pp. 1387-1395.

13. Konečný, J.; McMahan, H.B.; Yu, F.X.; Richtárik, P.; Suresh, A.T.; Bacon, D. Federated Learning: Strategies for Improving Communication Efficiency; NIPS Workshop on Private Multi-Party Machine Learning; NIPS: Barcelona, Spain, 2016.

14. Emam, K.E.; Dankar, F.K. Protecting privacy using k-anonymity. J. Am. Med. Inform. Assoc. 2008, 15, 627-637. [CrossRef] [PubMed]

15. Nergiz, M.E.; Clifton, C. $\delta$-presence without complete world knowledge. IEEE Trans. Knowl. Data Eng. 2010, $22,868-883$. [CrossRef]

16. Nikolaenko, V.; Weinsberg, U.; Ioannidis, S.; Joye, M.; Boneh, D.; Taft, N. Privacy-Preserving Ridge Regression on Hundreds of Millions of Records. In Proceedings of the IEEE Symposium on Security and Privacy, Berkeley, CA, USA, 19-22 May 2013; pp. 334-348.

17. Wan, J.; Wang, J.; Wang, Z.; Hua, Q. Artificial Intelligence for Cloud-Assisted Smart Factory. IEEE Access 2018, 6, 55419-55430. [CrossRef]

18. Federated Learning: Collaborative Machine Learning without Centralized Training Data (Google AI Blog). Available online: https: / / ai.googleblog.com/2017/04/federated-learning-collaborative.html (accessed on 15 April 2021).

19. Contreras, L.M.; Bernardos, C.J. Overview of Architectural Alternatives for the Integration of ETSI MEC Environments from Different Administrative Domains. Electronics 2020, 9, 1392. [CrossRef]

20. Yoon, Y.H.; Jang, S.Y.; Choi, D.Y.; Lee, S.E. Flexible Embedded AI System with High-speed Neuromorphic Controller. In Proceedings of the International SoC Design Conference (ISOCC), Jeju, Korea, 6-9 October 2019; pp. 265-266.

21. Yoon, Y.H.; Hwang, D.H.; Yang, J.H.; Lee, S.E. Intellino: Processor for Embedded Artificial Intelligence. Electronics 2020, 9 , 1169. [CrossRef]

22. Seng, K.P.; Lee, P.J.; Ang, L.M. Embedded Intelligence on FPGA: Survey, Applications and Challenges. Electronics 2021, 10, 895. [CrossRef]

23. Fujii, T.; Toi, T.; Tanaka, T.; Togawa, K.; Kitaoka, T.; Nishino, K.; Nakamura, N.; Nakahara, H. New Generation Dynamically Reconfigurable Processor Technology for Accelerating Embedded AI Applications. In Proceedings of the IEEE Symposium on VLSI Circuits, Honolulu, HI, USA, 18-22 June 2018; pp. 41-42.

24. Fang, F.; Shi, J.; Lu, S.; Zhang, M.; Ye, Z. An Efficient Computation Offloading Strategy with Mobile Edge Computing for IoT. Micromachines 2021, 12, 204. [CrossRef] [PubMed]

25. Lin, J.; Chen, W.M.; Lin, Y.; Cohn, J.; Gan, C.; Han, S. MCUNet: Tiny Deep Learning on IoT Devices. arXiv 2020, arXiv:2007.10319v2.

26. Banbury, C.; Zhou, C.; Fedorov, I.; Navarro, R.M.; Thakker, U.; Gope, D.; Reddi, V.J.; Mattina, M.; Whatmough, P.N. MicroNets: Neural Network Architectures for Deploying TinyML Applications on Commodity Microcontrollers. arXiv 2021, arXiv:2010.11267v6.

27. Burrello, A.; Garofalo, A.; Bruschi, N.; Tagliavini, G.; Rossi, D.; Conti, F. DORY: Automatic End-to-End Deployment of Real-World DNNs on Low-Cost IoT MCUs. IEEE Trans. Comput. 2021, 70, 1253-1268. [CrossRef]

28. Cubero, L.; Peizerat, A.; Morche, D.; Sicard, G. Smart imagers modeling and optimization framework for embedded AI applications. In Proceedings of the 15th Conference on Ph.D Research in Microelectronics and Electronics (PRIME), Lausanne, Switzerland, 15-18 July 2019; pp. 245-248.

29. Hwang, D.H.; Yoon, Y.H.; Han, C.Y.; Lee, S.E. Performance Analyzer for Embedded Artificial Intelligence Processor. J. Internet Comput. Serv. 2020, 21, 149-157.

30. Bouchhima, A.; Yoo, S.; Jerraya, A. Fast and accurate timed execution of high level embedded software using HW/SW interface simulation model. In Proceedings of the Asia and South Pacific Design Automation Conference, Yokohama, Japan, 27-30 January 2004; pp. 469-474.

31. General Vision Documents. Available online: https://www.general-vision.com/documentation/TM_CM1K_Hardware_Manual. pdf (accessed on 28 May 2021).

32. The MNIST DATABASE of Handwritten Digits. Available online: http://yann.lecun.com/exdb/mnist/ (accessed on 12 April 2021).

33. Warden, P. Speech Commands: A Dataset for Limited-Vocabulary Speech Recognition. arXiv 2018, arXiv:1804.03209. 